\title{
Brennpunkt Nadelstichverletzungen
}

\author{
Komplettumstellung auf sichere medizinische Instrumente gefordert
}

Verletzungen durch spitze oder scharfe Gegenstände sind eine tägliche Gefahr für Beschäftigte im Gesundheitswesen: Die Europäische Kommission schätzt die Anzahl in der EU auf 1,2 Millionen im Jahr mit einer weitaus höheren Dunkelziffer. Statistisch verletzt sich jeder Angestellte einmal im Jahr an Blutentnahmekanülen, Injektionsnadeln oder anderen medizinischen Instrumenten. Dabei kann es zur Übertragung bedrohlicher Erreger - wie Hepatitisviren oder HIV - kommen. Die Initiative SAFETY FIRST! Österreich hat sich zur Aufgabe gemacht, Nadelstichverletzungen entgegenzuwirken. Sie fordert die vollständige Umstellung aller betroffenen Institutionen auf so genannte Sichere Instrumente und Verfahren. Aktuell unterstützt SAFETY FIRST! Österreich die rasche Verabschiedung der Nadelstichverordnung durch das Bundesministerium für Arbeit, Soziales und Konsumentenschutz.

Die Allgemeine Unfallversicherungsanstalt (AUVA) verzeichnet im Schnitt 2.000 Nadelstichverletzungen jährlich für Österreich. Die Initiative SAFETY FIRST! Österreich schätzt die Anzahl der Nadelstichverletzungen jedoch auf das Achtfache, da viele Verletzungen vom medizinischen Personal - teils aus Gewohnheit, teils aus Scham - gar nicht gemeldet werden. Primäres Ziel der im vergangenen September gegründeten Initiative SAFETY FIRST! Österreich ist, medizinisches Personal nachhaltig vor Nadelstichverletzungen $\mathrm{zu}$ schützen. „Deshalb fordern wir die Komplettumstellung von konventionellen auf Sichere Instrumente in allen österreichischen Institutionen des Gesundheits-, Pflege- und Sozialwesens", bringt Leopold Karner, Hygienefachkraft am Landesklinikum Krems, das Hauptanliegen der Initiative auf den Punkt. SAFETY FIRST! wird vor allem intensive Informations- und Aufklärungsarbeit zum Thema Nadelstichverletzungen leisten. „Im Mittelpunkt steht für uns jedoch gegenwärtig die Verabschiedung der Nadelstichverordnung, zu der das Bundesministerium für Arbeit, Soziales und Konsumentenschutz kürzlich einen Begutachtungsentwurf veröffentlichte“, beschreibt Ing. Günter Pfaringer, Sicherheitsfachkraft bei der Tiroler
Landeskrankenanstalten $\mathrm{GmbH}$, die aktuelle Aufgabe der Initiative. „Wir haben dazu bereits beim Ministerium unsere Stellungnahme abgegeben und hoffen nun auf eine enge Zusammenarbeit", ergänzt Pfaringer.

Die Initiative SAFETY FIRST! Österreich möchte mit ihrer Expertise bei der Formulierung der „Verordnung zum Schutz der Arbeitnehmer/innen vor Verletzungen durch scharfe oder spitze medizinische Instrumente" (Nadelstichverordnung NastV) eng mit dem Bundesministerium für Arbeit, Soziales und Konsumentenschutz zusammen arbeiten. Dabei geht es um die fristgerechte Umsetzung der „EU-Richtlinie 2010/32/EU zur Vermeidung von Verletzungen durch scharfe/spitze Instrumente im Krankenhaus- und Gesundheitssektor" (siehe auch PROCARE 09/2011). Denn alle Mitgliedsstaaten müssen diese Richtlinie bis zum 11. Mai 2013 im nationalen Recht verankert und umgesetzt haben. „Wir begrüßen den Begutachtungsentwurf des Bundesministeriums für Arbeit, Soziales und Konsumentenschutz, möchten jedoch daran erinnern, dass wir uns bereits in der Übergangsphase befinden", betont Dr. Stephan Konzett, Arbeitsmediziner am Landeskrankenhaus Feldkirch und Hygienebeauftragter Arzt, die Dringlichkeit der Umstellung auf Sichere Instrumente.

SAFETY FIRST! Österreich lädt alle betroffenen Institutionen, Entscheider und Berufsgruppen ein, gemeinsam Projekte für den bestmöglichen Schutz der österreichischen Arbeitnehmerinnen und Arbeitnehmer im Gesundheits-, Pflege- und Sozialwesen vor Nadelstichverletzungen umzusetzen. Dabei geht es unter anderem um die Erörterung der Qualitätskriterien Sicherer Instrumente, das Erstellen einer Best-Practice-Leitlinie für die Umstellungsphase sowie die Begleitung medizinischen Personals bei der Anwendung der neuen Instrumente, zum Beispiel durch intensive Schulungen.

\section{Mit dem Tablet-PC zum Patientenbett}

\section{Schmerzen nach Operationen besser in den Griff bekommen}

So gut und erfolgreich eine Operation auch verlaufen sein mag, Schmerzen können nach einer OP bei jedem Patienten auftreten. Daher sollten für jeden Patienten nach einer OP eine Schmerzerhebung und eine individuell abgestimmte Schmerztherapie erfolgen. Die Anästhesisten im Krankenhaus der Barmherzigen Schwestern in Linz und Wien dokumentieren das Schmerzempfinden der operierten Patienten mobil mittels Tablet-PC. Die Daten werden zum Zentralcomputer geschickt und damit sofort zur Patientenakte gespeichert. Weitere Spitäler der Vinzenz Gruppe werden diesen Ansatz teilweise noch heuer übernehmen.

Dieses Konzept stand auch bei den diesjährigen Anästhesie-Kongresstagen der Vinzenz Gruppe Ende November im Orthopädischen Spital Speising in Wien auf dem Programm. „Die postoperative Schmerztherapie hilft uns auch, ökonomischer zu wirtschaften", erklärte Dr. Johann Blasl, Leitender Anästhesist im Krankenhaus Barmherzige Schwestern Wien: „Denn durch die Dokumentation und Behandlung der Schmerzen können viele Patienten - zum Beispiel nach einer Gelenksersatz-Operation - schon viel früher mobilisiert werden, sprich: das Krankenhaus verlassen. Aktuelle Studien zeigen, dass man dadurch dem Gesundheitssystem bis zu 1.500 EUR pro Patient ersparen kann“.

Ein weiteres zentrales Thema am Anästhesie-Kongress widmete sich der Ethik in der Anästhesie. Am Rande des Symposiums diskutierten die Experten auch über Hypnose in der Anästhesie: „Sie kann ein Bestandteil der Narkose sein“, erläuterte Blasl.

Quelle: Presseaussendung, Vinzenz Gruppe Krankenhausbeteiligungs- und Management $\mathrm{GmbH}$, Wien 